\title{
Early Detection and Prevention of Neonatal Sepsis
}

Ketevan Nemsadze

Additional information is available at the end of the chapter

http://dx.doi.org/10.5772/56121

\section{Introduction}

Sepsis has been a burden to mankind for millions of years and will continue to plague man as long as microorganisms exist here on earth. Only recently the medical community has started celebrating the World Sepsis Day (WSD) which was established in 2012, yet a decade before, at the end of the $20^{\text {th }}$ century the Anti-Sepsis Center was founded in Georgia. [1]

Research shows that early recognition and intervention saves lives. To achieve this improvement requires a partnership between the public, parents, and healthcare professions. Sepsis is a common pediatric problem. Severe sepsis and septic shock are among the leading causes of death in infants and have an overall pediatric mortality rate of $8-10 \%$. Definitive diagnosis requires clinical identification of infection in a patient who also meets the clinical criteria for the Systemic Inflammatory Response Syndrome (SIRS). [2]

In the given chapter, early recognition, diagnostic criteria, treatment and prevention of neonatal sepsis are described.

\section{Definition}

Neonatal Sepsis - is a clinical syndrome which is a general reaction to infection. Neonatal sepsis is characterized by systemic inflammation and general damage of tissues. Clinical definition is based on existing infection and systemic inflammatory response. Neonatal sepsis is diagnosed on the basis of clinical or microbiological data. Neonatal sepsis is an irreversible process which may cause mortality in cases of untimely detection and treatment. [3] 


\section{Terminology}

Frequently Used Terms Referring to Sepsis: Neonatal Fever; Neonatal Sepsis; Serious Bacterial Infection (SBI); Systemic Inflammatory Response Syndrome (SIRS); Septic Shock (= Sepsis + Cardiovascular dysfunction).[2]

\section{Classifications of Neonatal Sepsis}

Neonatal and infant sepsis is classified according to age at the time of disease manifestation.

'EARLY' SEPSIS_manifests within the first 72 hours after birth by the vertical transfer of microorganisms existing in maternal passages. It is characterized by fulminant multiple organ damage. Symptoms of pneumonia may be revealed within the first week of life.

'EARLY, EARLY' SEPSIS AND VERY EARLY SEPSIS manifests within the first 24 hours after birth by the vertical transfer of microorganisms existing in maternal passages.

'LATE' SEPSIS manifests within the first 72 hours after birth by the vertical or horizontal transfer of microorganisms existing in maternal passages. The primary cause of late sepsis is hospital infection. It is characterized by gradual development and multiple nidus of infection. Meningitis can occur quite frequently. Sepsis may manifest within the first 3 months of the child's life.

LATE, LATE' SEPSIS OR VERY LATE SEPSIS manifests more than 3 months after birth mainly in children born before 28 weeks of pregnancy or with immunodeficiency.[4,3]

\section{Frequency of Early Sepsis of Neonates (Tbilisi Central Children's hospital)}

Full-Term Infants: 0.2\% (among 2/1000 neonates)

Late pre-term infant: $0.3 \%$ (among $3 / 1000$ neonates)

Low Birth-Weight Infants: 1.5\% (15/1000 neonates)

Very Low Birth-Weight Infants: 2.5\% (25/1000 neonates)

Extremely Low Birth-Weight Infants: 25\% (250/1000 neonates)

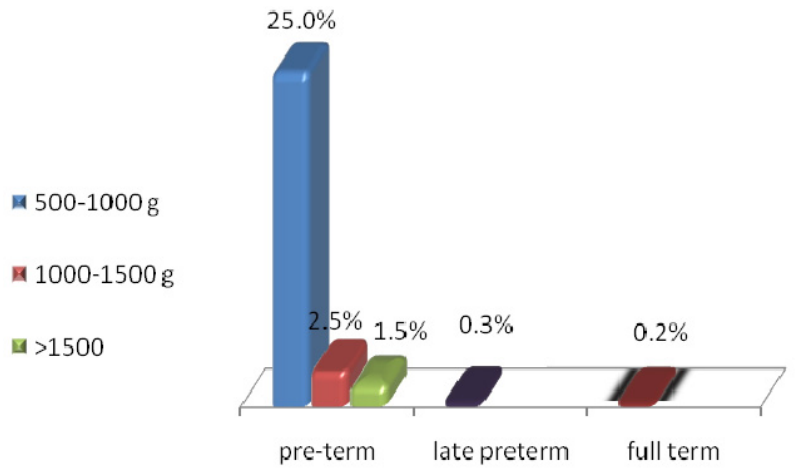

Figure 1. K. Nemsadze, Neonatology, 2010 [1] 
The frequency of early sepsis in infants with extremely low-birth-weight is 100 times higher than in full-term babies. Considering the fact that the number of children born at a gestational age greater than 35 weeks is much more common, the data shows that neonates with low birth weight are far more likely to be diagnosed with sepsis. [1]

\section{Frequency of Late Sepsis of Neonates}

Late Sepsis is predominantly nosocomial (hospital disease) thought in some cases infection may be connected with maternal organisms. This form of clinical sepsis is one of main clinical problems characterized primarily by significantly premature babies. Development of late sepsis in neonates of this group is associated with a significant increase in the frequency of complications, mortality and the prolonged hospitalization of neonates.

In West Europe, North America and Australia - late sepsis frequency is up to 6 among 1000 neonates. Among neonates of gestational age less of 25 weeks, late sepsis develops in $46 \%$ of them; among neonates of gestational age between 25-28 weeks, late sepsis develops in $29 \%$. Thus, the less gestational age the higher the probability of developing late sepsis. The frequency of nosocomial infections is inversely proportional to birth weight and gestational age of neonates. This complication cannot only be explained by the prolonged hospitalization needs of extremely premature children. [5]

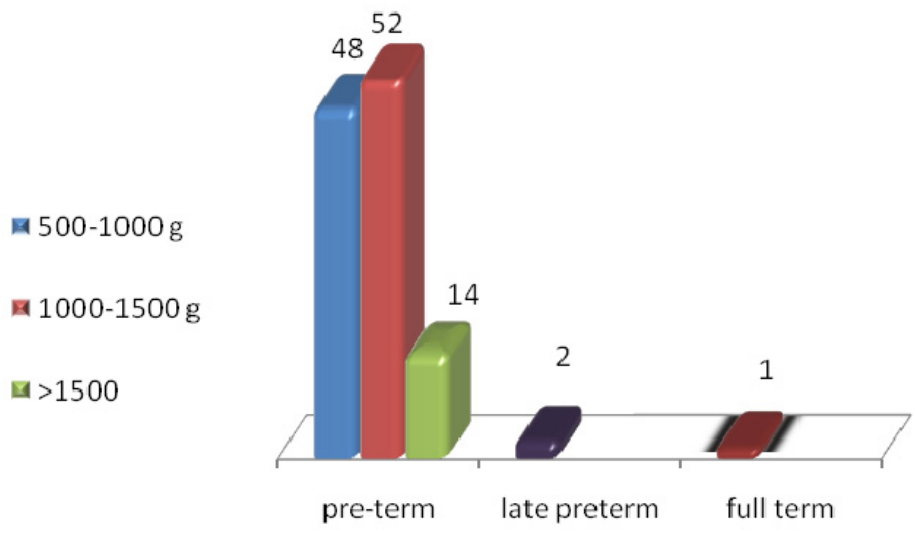

Figure 2. K. Nemsadze, Neonatology, 2010 [1] 


\section{Etiology and epidemiology}

Etiological Structure of Sepsis in Developed Countries

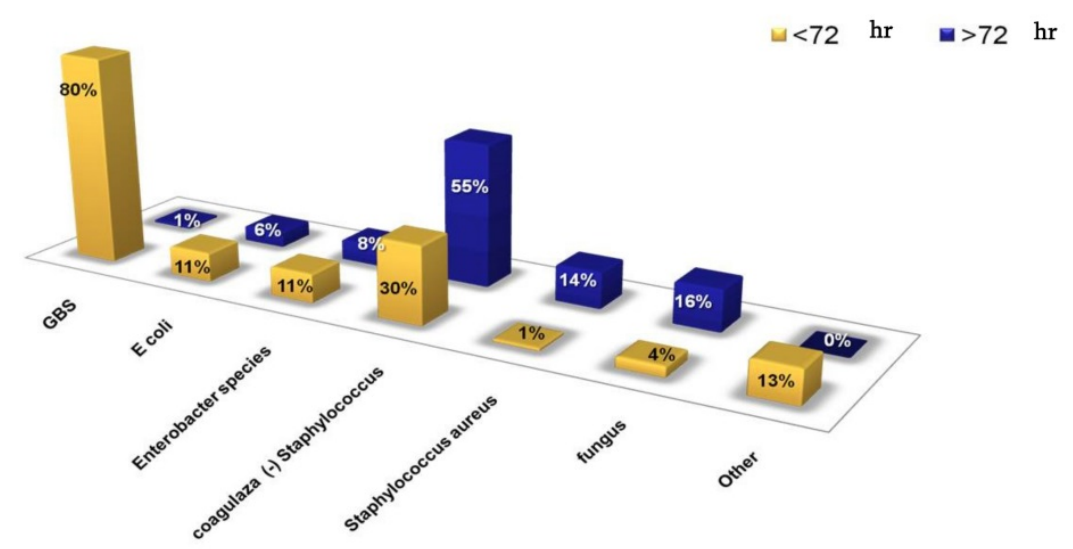

Figure 3. J. Garcia-Prats et al.,SeminPediatrInfect Dis, 2000;11:4 [6]

\section{Etiological Structure of Sepsis in Tbilisi Children's Central Hospital}

Consequently, the main causative agents of neonatal sepsis are bacteria such as staphylococcus.

Klebsiella, acinetobacter and staphylococcus aureus can be the cause of early as well as late sepsis

Late sepsis is usually a nosocomial disease although is some cases it may be connected with vertical infection. Pseudomonas, salmonella and serratia can often cause late sepsis. Many factors impact the etiological structure, including the quality of life, cultural traditions, practice of antibiotic therapy and the possibility of distorted results caused by many neonates to die at home.

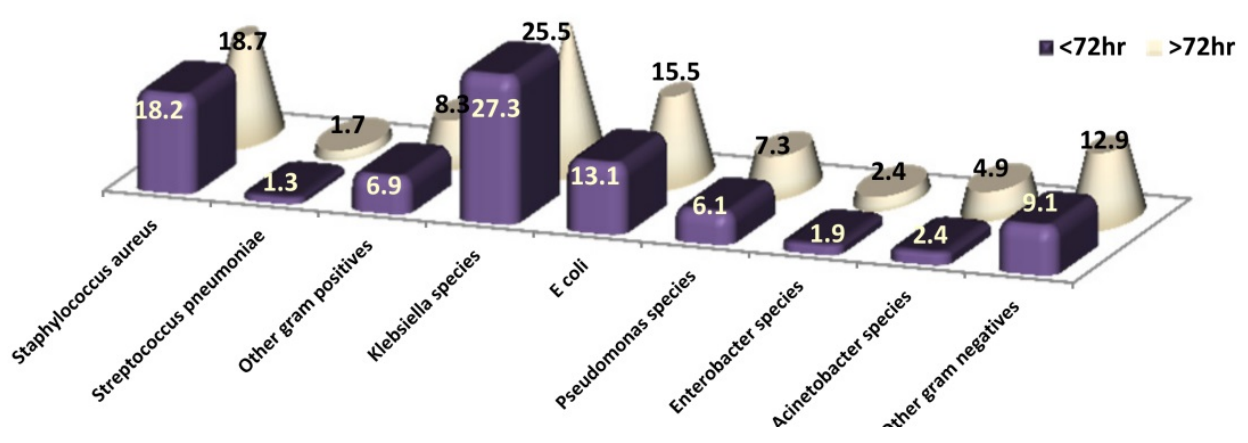

Figure 4. K. Nemsadze, Neonatology, 2010 [1] 


\section{Pathophysiology}

In the presence of sepsis, the response of infection released anti-inflammatory mediators can't localize anti-inflammatory process. Generalized infection is formed as sepsis. The cause of sepsis (SIRS) is multi-factorial: Activation of anti-inflammatory mediators; complement ischemia of tissues; cytopathology; changes in apoptosis rate. Cellular damage with discharge of anti-inflammators (IL1, IL6 TNF- Tumor Necrosis Factor) and antiinflammatory mediators increase the probability of developing multi-organ failure. The cardiovascular system, pulmonary system, gastro-intestinal tract, kidneys and neurological system are most frequently damaged. Given mediators stimulate production of various proteins called as reagents of acute phase. Any kinds of inflammation stimulus, including infection, trauma and ischemia causes marginal extravasations and activation of granulocytes and monocytes with the simultaneous release of anti-inflammatory cytokines including interleukins IL1, IL6 TNF (Tumor Necrosis Factor) [7]

\section{Sepsis is caused by Systemic Inflammatory Response (SIR)}

It is widely known that sepsis educes as a result of Systemic Inflammatory Response (SIR). In the process of opsonization and macrophage phagocytosis pathogens cause the formation of various anti-inflammatory mediators (cytokines) which damage vessel endothelium the result of which is the release of tissue factors. The coagulation system is activated and fibrinolysis inhibitor activity is increased. [8,9]

\section{Anti-inflammatory Mediators are Reagents of the Acute Phase}

After the patient is stabilized, normalization and a secondary increase in the levels of CBC indicate the sepsis complication (subdural empyema and bacterial meningitis [10]. Sepsis may change the metabolism of neonates. The change in metabolism may be described in two phases

$$
\text { Ebb Phase } \rightarrow \text { Flow Phase }
$$

\section{EBB Phase}

The initial EBB phase lasts 1-3 days. In this phase the neonate is at the stage of compensation while metabolism is slowed.

\section{EBB Phase Consists of Some Clinical Symptoms:}

- Hypometabolism

- Decrease of energy consumption

- Reduction of cardiac output

- Hypoxia

- Vasoconstriction

\section{Flow Phase}

Flow Phase follows the initial Ebb Phase. In this phase the organism goes into a hyperactive state, which is particularly conditioned by a hyper inflammatory reaction. In many cases flow phase leads to patient mortality. 
Flow Phase Includes Some Clinical Symptoms:

- Hypermetabolism

- Increase in energy consumption

- Increase in cardiac output

- Hyperoxia

\section{Risk factors}

\section{Maternal Premature Birth Risk Factors}

- Preterm rupture of the fetus membrane

- Anhydrous periodgreater than 18 hours

- Febrile temperature greater than $38^{\circ} \mathrm{C}$ (at or after birth)

- GBS bacteriuria during current pregnancy (>104 cfu/mL)

- GBS bacterial colonization of the vagina/perineum

- Chorioamnionitis/Endometritis

- Infection of the Urino-Genital Systems

- Invasive Procedures

- Previous child with GBS infection

- Previous child born with sepsis

- Multiple pregnancy

Neonatal Risk Factors

- Premature neonate less than 37 weeks of gestation, Low birth weight and Small for Gestational Age(SGA)

- Male sex

- Stable intranatal fetal tachycardia

- Asphyxia/Resuscitation

- Hypothermia

- Invasive procedures

- Artificial feeding

- Non-insurance fetus

- Lack of "Skin-to-skin" contact with mother

- Long-term Hospitalization; Irrational antibiotic therapy

- Poor sanitary habits of medical personnel.

It is essential to know the evidence-based risk factors of neonatal infection as modern strategies of prophylaxis are precisely based on this data. [11]

\section{Change of Body Temperature as a Sign of Possible Infection in Neonates}

The use of a mercury glass thermometer is considered the gold standard for measuring the normal body temperature of neonates - 36.5 - 37.5 C. Sepsis is characterized by arise in temperature or hypothermia. Rise of temperature greater than $38^{\circ} \mathrm{C}$ that lasts more than an hour is associated with infection process.[12] 
On the analysis of their research, Klein and Marcy made the following conclusions referring to diagnostic analysis of symptoms and the relation to rising body temperature in full-term neonates: If bodily temperature is less than $38^{\circ} \mathrm{C}, 99.9 \%$ of neonates will not develop sepsis. When among neonates with body temperature more than $38{ }^{\circ} \mathrm{C}$, only $10 \%$ are expected to developed sepsis.

\section{Clinical symptomatology}

Nonspecific clinical signs: (3P-Signs)

- Poor breathing

- Poor sucking

- Poor looking

Clinical signs are various and the diversity of symptoms is the result of metabolic and inflammatory processes arisen in case of neonatal sepsis. [12]

Consequently, clinical symptoms of sepsis are nonspecific: They cannot be summarized according to principles: Poor Breathing, Poor Eating and Poor Looking. Out of 10 children with suspected sepsis, the disease will be confirmed in only one.

\section{Symptoms of Respiratory Disorders:}

- Tachypnea (>60 in min)

- Chest retraction

- Grunting while breathing

- Inflating the nostrils (nose wings)

- Apnea/bradypnea (<30 in a min)

- Hypoxia

- Irregular breathing [12]

Symptoms of Gastro-intestinal and Neurologic Disorders:

- Gastro-intestinal

- Loss of appetite

- Vomiting; Diarrhea

- Abdominal distension

- Splenomegaly

- Neurologic

- Convulsions

- Hypotonia and Hypodynamia

- Lethargy [12]

Symptoms of Cardiovascular and Skin Disorders:

\section{Cardiovascular:}

- Hypotension 
- Metabolic Acidosis

- Tachycardia

Skin:

- Pale or marble with petechia or purple

- Mottling

- Cold or wet

- Cyanosis

- Jaundice [12]

\section{Evaluations criteria of neonatal sepsis}

Bacterial inoculation of blood; Leukocytes $\left(<5\right.$ or $\left.>30 \times 10^{9} / 1\right)$; Total number of neutrophils; Leukocyte index (LI) >0,2; CRP $>10 \mathrm{mg} / \mathrm{l}$ (Initial examination no later than 12 hours from birth); ESR $>15 \mathrm{~mm} / \mathrm{hr}$; Chest X-ray in the presence of Respiratory Distress Syndrome (RDS); Lumbar puncture in the presence of neurological symptoms; Bacterial inoculation of urine by using catheter or suprapubic puncture. [13]

Lumbar Puncture (LP): When should a lumbar puncture be conducted?

Symptoms of sepsis and any of the following symptoms;

Bulging of fontanel, any neurological symptoms, leukocytes $<5$ or $>30 \times 109$ in 24 hours or $>$ 20 x 109 since second 24 hours or Leukocyte Index (LI) $>0,4$ or CRP $>40 \mathrm{mg} / \mathrm{l}$ or $>2$, laboratory Indexes with obvious abnormality.[13]

\section{Evaluation Criteria of Late Sepsis}

Common clinical conditions if sepsis is suspected; Instability of body temperature; Gastrointestinal symptoms (vomiting, abdominal distension, blood in stool, increase in quantity of residual mass in stomach); Neurological symptoms; Cardiorespiratory dysfunction $(100<\mathrm{HR}>180,30<\mathrm{BR}>60$, hypotension, time of capillary filling $>4 \mathrm{sec})$; Respiratory symptoms (toughening of parameters of lungs mechanical ventilation of BR $>60$, apnoea); Metabolic acidosis; hyperglycemia/hypoglycemia; Leukocytosis; leukopenia; Ratio of immature neutrophils compared to the total number of neutrophils (LI)>2,0 , thrombocytopenia

Treatment should starts in case of possible sepsis if;the mother is suspected of having infection; the infant has clinical signs of infection; the infant has possible signs of infection in combination with low weight at birth, asphyxia and other risk factors; positive results of screening tests and/or bacterial investigation.

\section{Indication of prophylaxis usage of antibiotics during delivery}

GBS bacteruria during pregnancy; Previous child with GBS infection; Mother's temperature greater than $38^{\circ} \mathrm{C}$, even when GBS culture is negative; Unknown GBS status and early 
discharge of amniotic fluid $>18 \mathrm{hr}$, gestational age less than 37 weeks, mother's temperature is greater than $38^{\circ} \mathrm{C}$. [2]

\section{Antenatal Prophylaxis}

It is important to use a wide variety of laboratory methods for identification of GBS (Group $B$ streptococcus). Defining the number of colonies in urine of pregnant women is necessary to diagnose GBS.[2]

Main changes in the 2010 guidelines include the following: Intranatal Prophylaxis - Change of penicillin-G recommended dose for chemoprophylaxis; Updating schemes of prophylaxis for women with an allergy to penicillin and revising the algorithms. [2]

Secondary Prophylaxis of GBS in Case of Early Sepsis of Neonates

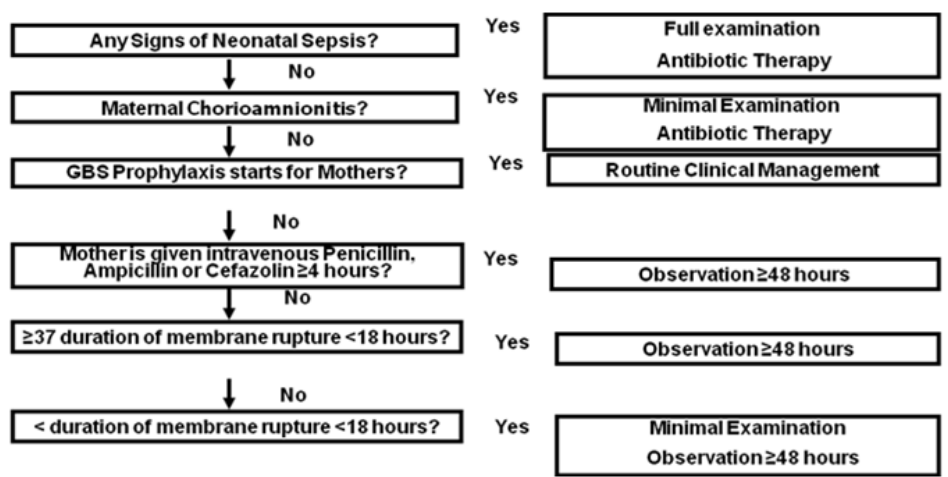

Figure 5. Prevention of Perinatal Group B Streptococcal Disease Revised Guidelines from CDC, 2010 [11]

Full diagnostic evaluation includes a blood culture, a complete blood count (CBC) including white blood cell differential and platelet counts, chest radiograph (if respiratory abnormalities are present), and lumbar puncture (if patient is stable enough to tolerate procedure and sepsis is suspected).Antibiotic therapy should be directed toward the most common causes of neonatal sepsis, including intravenous ampicillin for GBS and coverage for other organisms (including Escherichia coli and other gram-negative pathogens) and should take into account local antibiotic resistance patterns. Consultation with obstetric providers is important to determine the level of clinical suspicion for chorioamnionitis. Chorioamnionitis is diagnosed clinically and some of the signs are nonspecific. Limited evaluation includes blood culture (at birth) and CBC with differential and platelets (at birth and/or at 6-12 hours of life). If signs of sepsis develop, a full diagnostic evaluation should be conducted and antibiotic therapy initiated. If 37 weeks' gestation, observation may occur at home after 24 hours if other discharge criteria have been met, access to medical care is readily available and a person who is able to comply fully with instructions for home observation will be present. If any of these conditions are not met, the infant should be observed in the hospital for at least 48 hours and until discharge criteria are achieved. Some experts recommend a CBC with differential and platelets at age 6-12 hours. [11] 


\section{Principles of treatment}

Early Sepsis - Ampicillin + Gentamicin; Late Sepsis - Cefotaxime+ Aminoglycosides; Consequent A/B therapy depending on the results of repeated blood inoculation Vancomycin and/or Meropenem and/or Antimycotic Drugs.

It's important to consider local epidemiological/microbiological data.

Empyreal antibiotic therapy of early sepsis must impact on gram negative and gram positive microorganisms. It's important to remember that listerias are potential agents for early infection of neonates. It is necessary to prescribe 2 antibiotics which cover a wide enough spectrum and at the same time resist a selection of antibiotic resistant bacteria. In case of late hospital sepsis it is particularly important to affect staphylococcus and gram negative bacteria. If mother discharges GBS during delivery it is advised that penicillin be prescribed.

NB! Cephalosporin is ineffective towards enterococcus and listerias!

Figure 6. Therapeutic guidelines in neonatal infection 2011[11]

\section{Duration of Antibacterial Therapy}

Absence of clinical symptoms and negative results of investigation: 48-72 hr; In case of gram + flora - 7 or more days; In case of gram + flora - minimum 14 days; In case of meningitis 21 days; Consequent a/b therapy should depend on the results of repeated blood culture investigations.[12]

The duration of antibacterial therapy depends on clinical form of infection while therapy of osteomyelitis/endocarditis it is recommended to change antibiotics only in case of absence of effect of conducted therapy.

\section{Dosage of Antibiotics: Ampicilin}

AMPICILIN - Single dose 25-50 mg/kg IntraV/IntraM GBS Infection.

In cases of bacteria it is permitted- $150-200 \mathrm{mg} / \mathrm{kg} /$ day;

In cases of meningitis- $300-400 \mathrm{mg} / \mathrm{kg}$ in a day

\begin{tabular}{|c|c|c|}
\hline Gestational Age (weeks.) & Child Age (days) & Interval between Injection (hours) \\
\hline \multirow{2}{*}{$\leq 29$} & $0-28$ & 12 \\
& $>28$ & 8 \\
\hline \multirow{2}{*}{$30-36$} & $0-14$ & 12 \\
& $>14$ & 12 \\
\multirow{2}{*}{$37-44$} & $0-7$ & 8 \\
\hline \multirow{2}{*}{$\geq 45$} & $>7$ & 6 \\
\hline
\end{tabular}

Table 1. Therapeutic guidelines in neonatal infection 2011 [11]

Neofax 2009 Twenty Second Edition [14] 


\section{Dosage of Antibiotics: Gentamicin}

\section{GENTAMICIN}

Injected intravenously slowly during 30 minutes.

\begin{tabular}{|c|c|c|c|}
\hline $\begin{array}{c}\text { Gestational Age } \\
\text { (weeks.) }\end{array}$ & Postnatal Age (days) & Dose (mg/kg) & $\begin{array}{c}\text { Interval between } \\
\text { Injection (hours) }\end{array}$ \\
\hline \multirow{3}{*}{$\leq 29$} & $0-7$ & 5 & 48 \\
& $8-28$ & 4 & 34 \\
$\geq 29$ & 4 & 24 \\
\hline \multirow{2}{*}{$30-34$} & $0-7$ & 4,5 & 36 \\
& $\geq 8$ & 4 & 24 \\
\hline$\geq 35$ & All & 4 & 24 \\
\hline
\end{tabular}

Table 2. Therapeutic guidelines in neonatal infection 2011 [11]

Neofax 2009 Twenty Second Edition [14]

Dosage of Antibiotics: Amikacin

\section{AMIKACIN}

Injected intravenously slowly during 30 minutes.

\begin{tabular}{|c|c|c|c|}
\hline $\begin{array}{c}\text { Gestational Age } \\
\text { (weeks.) }\end{array}$ & $\begin{array}{c}\text { Postnatal Age } \\
\text { (days) }\end{array}$ & Dose (mg/kg) & $\begin{array}{c}\text { Interval between } \\
\text { Injection (hours) }\end{array}$ \\
\hline \multirow{3}{*}{29} & $0-7$ & 18 & 48 \\
& $8-28$ & 15 & 36 \\
\hline \multirow{2}{*}{$30-34$} & $\geq 29$ & 15 & 24 \\
\hline$\geq 35$ & $0-7$ & 18 & 36 \\
\hline & $\geq 8$ & 15 & 24 \\
\hline
\end{tabular}

Table 3. Therapeutic guidelines in neonatal infection 2011 [11]

Neofax 2009 Twenty Second Edition [14,15] 


\section{Dosage of Antibiotics: Cefotaxime}

\section{CEFOTAXIME}

Single Dose $50 \mathrm{mg} / \mathrm{kg}$ intravenously slowly during 30 minutes Or I/M

The dosage of antibiotics is relevant to the etiological agent and depends on gestational and postnatal age of the neonate and it is chosen according to the Neofax guide.[14]

\begin{tabular}{|c|c|c|}
\hline $\begin{array}{c}\text { Gestational Age } \\
\text { (weeks.) }\end{array}$ & $\begin{array}{c}\text { Postnatal Age } \\
\text { (days) }\end{array}$ & $\begin{array}{c}\text { Interval between Injection } \\
\text { (hours) }\end{array}$ \\
\hline$\leq 29$ & $0-28$ & 12 \\
& $>28$ & 12 \\
\hline \multirow{2}{*}{$30-36$} & $0-14$ & 8 \\
\hline \multirow{2}{*}{$37-44$} & $>14$ & 12 \\
& $0-7$ & 8 \\
\hline 45 & $>7$ & 6 \\
\hline
\end{tabular}

Table 4. Therapeutic guidelines in neonatal infection 2011[11]

Neofax 2009 Twenty Second Edition [14]

\section{Consultation with parents}

Complications include the following: Septic shock; Necrotic tonsillitis enterocolitis, ulceronecrotic enterocolitis; Subdural empyema; Meningitis

\section{Information for Patients}

\section{What is Sepsis?}

Sepsis - Is the existence of infection in the blood. Sepsis is a serious disease that impacts the whole body. Treatment of Sepsis should be started immediately after diagnosis, because the late start of treatment may endanger life. Sepsis may occur in infants, children and adults as well. Sepsis diagnosed in children less than 1 month old is specified by the term "Sepsis of Newborn".

\section{Definition}

Neonatal sepsis is a clinical syndrome which represents a general reaction to infection. It is characterized by systemic inflammation and general damage of tissues. Clinical definition is based on existing infection and systemic inflammatory response. Neonatal 
sepsis is diagnosed on the bases of clinical or microbiological data. Neonatal sepsis is an irreversible process which may cause mortality in cases of untimely detection and treatment.

\section{Which symptoms are specific for sepsis in newborns?}

- Fever, though some children may have low or normal body temperature

- Breathing problems or fast heart rate

- Baby feeds poorly

- Vomiting

- Jaundice (baby's skin or white tunic of eyes turn a yellowish tinge)

- Somnolence (difficulty in waking the child)

- $\quad$ Fingers and lips remain cyanotic (blue or purple coloration in the skin)

Danger signs:

When is it necessary to contact the doctor?

You should contact your pediatrician if your baby has one of above-listed symptoms or looks sick.

\section{Is there a need to conduct laboratory tests on a child?}

Yes, the doctor will ask: About the symptoms the baby has, previous deliveries and the baby's status at birth. Observation of child will be conducted and blood analysis of the child will be done including tests known as 'Blood Inoculation'. These tests may determine the existence of infection in the blood.

Frequently, there is need to conduct further laboratory testing in order to determine the existence of infection in different parts of body. Examples of some of these tests may be:

Lumbar Puncture (During this procedure doctor inject a thin needle into the lower part of backbone to obtain a small amount of spinal fluid. Lumbar liquid helps diagnose disease in the brain and spinal cord), analysis of urine, X-ray of thorax.

What kind of treatment is conducted for newborns with sepsis?

Most treatment is conducted in hospitals. Doctor will prescribe antibiotics (drug against infection) for your baby. The drug is given intravenously via a tube called an 'IV'.

\section{Algorithm of management for suspected neonatal sepsis}

Note: $1 \mathrm{ml}$. is sufficient for bacterial analysis of blood if a pediatric bottle is used. All material will be used for aerobic culture therefore anaerobic organisms rarely cause early neonatal sepsis. If there is a catheter, blood is obtained simultaneously from the central and peripheral catheter.

It is desirable that diagnostic tests be repeated 24 hours after the first examination. [11] 


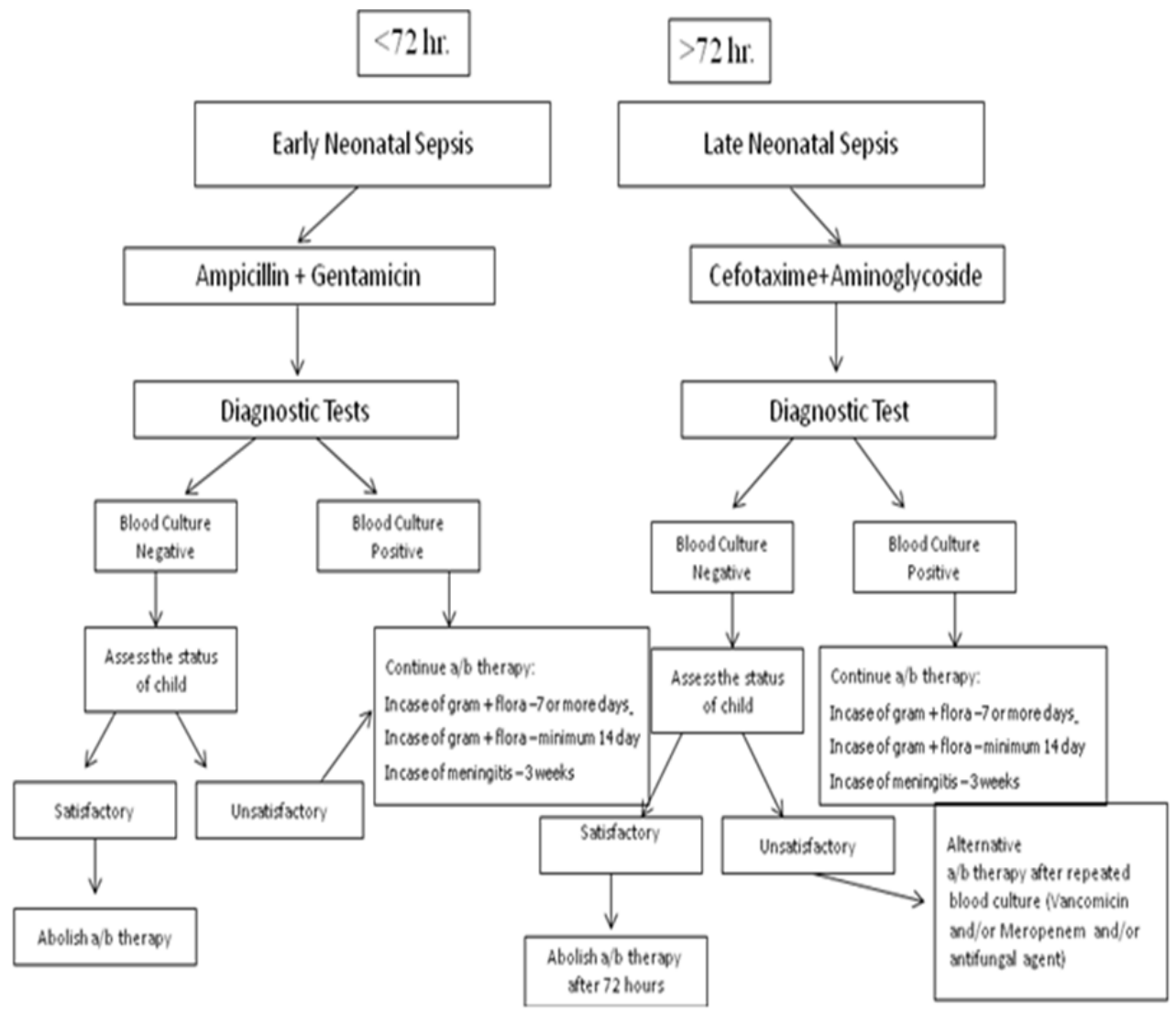

Figure 7. Therapeutic guidelines in neonatal infection 2011[11]

\section{Conclusions and recommendations}

1. Neonatal sepsis requires the immediate diagnosis and treatment in neonates regardless of gestational age or body weight at birth.

2. It is classified as early $(<72 \mathrm{hr})$ and late $(>72 \mathrm{hr})$; Late-late sepsis or Very Late Sepsis developing after 3 months of life in premature neonates with immune deficiencies.

3. Group B streptococcus (GBS) and staphylococcus are the most frequent agents of neonatal sepsis.

4. Risk factors of neonatal sepsis include GBS bacteriuria at ongoing pregnancy $(>104 \mathrm{cfu} / \mathrm{mL})$, colonization of mothers by GBS, duration of anhydrous interval $\geq 18$ hours, mother's temperature at delivery $\geq 38^{\circ}$.

5. Non-specific and various clinical symptoms.

6. Evaluation of neonates if sepsis is suspected, must include perinatal anamnesis, full physical examination and laboratory tests including CBA with leukogram, blood culture, lumbar puncture for the exception of meningitis before $a / b$ therapy starts, 
culture of urine when the age of neonate is at least 6 days old and a culture obtained from other possible infections of nidus.

7. Differential diagnosis of sepsis must be conducted with other suspected systemic infections, neonatal hypoxia, congenital metabolic abnormalities of metabolism and neonatal respiratory distress.

Despites the fact that full-term and premature neonates acquiring sepsis is low, the possibility of serious consequences including death is so high that there is the need to conduct an immediate diagnoses and treatment of possible sepsis in neonates regardless of gestational age or body weight at birth.

\section{Author details}

Ketevan Nemsadze

Georgian National Academy of Sciences, Georgia

\section{References}

[1] Nemsadze K. "Neonatology", 2010

[2] Micah Bhatti, Alison Chu, Joseph R. Hageman, Michael Schreiber and Kenneth Alexander: "Future Directions in the Evaluation and Management of Neonatal Sepsis"; Neoreviews 2012;13;e103 DOI: 10.1542/neo.13-2-e103

http://neoreviews.aappublications.org/content/13/2/e103

[3] Jennifer R. Verani, MD, Lesley McGee, PhD, Stephanie J. Schrag, DPhil: Centers for Disease Control and Prevention (CDC): "Prevention of Perinatal Group B Streptococcal Disease RevisedGuidelines(GBS)":

http://www.cdc.gov/groupbstrep/guidelines/new-differences.html (2010)

[4] Hammad A. Ganatra, MBBSa, Barbara J. Stoll, MDb,Anita K.M. Zaidi, MBBS,Sma "International Perspective on Early-Onset Neonatal Sepsis"; 2010

[5] Stoll BJ, Hansen N, Fanaroff AA, et al. Late-onset sepsis in very low birth weight neonates: the experience of the NICHD neonatal research network. Pediatrics. 2002;110:285-291.

[6] J. Garcia-Prats et al., Semin"Pediatric Infectional Disease", 20 00;11:4

[7] Hotchsepsis. Department of Anestkiss RS, Karl IE, The pathophysiology and treatment of hesiology, Washington University School of Medicine, 2003

[8] Sharma, S. \& Mink, S. (2004). Emedicine: "Septic Shock". http://www.emedicine.com/MED/topic2101.htm (Accessed February 14, 2006)

[9] Gabay C, Kushner I. "Acute-phase proteins and other systemic responses to inflammation". New England Journal of Medicine; 1999;340(6):448-4; with permission

[10] Orr, P.A., Case, K.O., \& Stevenson, J..J. "Metabolic response and parenteral nutrition in trauma sepsis and burns Journal of Infusion Nursing"; 2002. 25(1), 45-53. Retrieved March 7, 2006 from Ovid database 
[11] "Therapeutic guidelines in neonatal infection".2011

[12] J. Klein, S. Marcy. "Bacterial sepsis and meningitis". Mosby, 1995

[13] William E. Benitz, MAdjunct." Laboratory Tests in the Diagnosisof Early-OnsetNeonatal Sepsis";2009

[14] Neofax2009 Twenty Second Edition

[15] Epidemiology and Diagnosis of Health Care-Associated Infections in the NICU, the Committee on Fetus and Newborn and the Committee on Pediatrics 2012;129;e1104 Infectious Diseases DOI: 10.1542/peds.2012-0147; http://pediatrics.aappublications.org/content/129/4/e1104.full.html

[16] Morven S Edwards, MD “Clinical features and diagnosis of sepsis in term and late preterm infants" updated: Dec 18, 2012 http://www.uptodate.com/contents/clinicalfeatures-and-diagnosis-of-sepsis-in-term 